\title{
Local Power For Combining Independent Tests in The Presence of Nuisance Parameters For The Logistic Distribution
}

Walid A. Abu-Dayyeh

Yarmouk University, Irbid Jordan, abudayyehw@yahoo.com

Z.R.Al-Rawi

Yarmouk University Irbid-Jordan

M.M.A.Al-Momani

Yarmouk University Irbid-Jordan

Follow this and additional works at: http://digitalcommons.wayne.edu/jmasm

Part of the Applied Statistics Commons, Social and Behavioral Sciences Commons, and the Statistical Theory Commons

\section{Recommended Citation}

Abu-Dayyeh, Walid A.; Al-Rawi, Z. R.; and Al-Momani, M. M. A. (2005) "Local Power For Combining Independent Tests in The Presence of Nuisance Parameters For The Logistic Distribution," Journal of Modern Applied Statistical Methods: Vol. 4 : Iss. 1 , Article 8. DOI: $10.22237 /$ jmasm/1114906080

Available at: http://digitalcommons.wayne.edu/jmasm/vol4/iss1/8

This Regular Article is brought to you for free and open access by the Open Access Journals at DigitalCommons@WayneState. It has been accepted for inclusion in Journal of Modern Applied Statistical Methods by an authorized editor of DigitalCommons@WayneState. 


\title{
Local Power For Combining Independent Tests in The Presence of Nuisance Parameters For The Logistic Distribution
}

\author{
W. A. Abu-Dayyeh Z. R. Al-Rawi M. MA. Al-Momani \\ Department of Statistics, Faculty of Science \\ Yarmouk University Irbid-Jordan
}

Four combination methods of independent tests for testing a simple hypothesis versus one-sided alternative are considered viz. Fisher, the logistic, the sum of P-values and the inverse normal method in case of logistic distribution. These methods are compared via local power in the presence of nuisance parameters for some values of $\alpha$ using simple random sample.

Key words: combination method; independent tests; logistic distribution; local power; simple random sample; nuisance parameter.

\section{Introduction}

Combining independent tests of hypotheses is an important and popular statistical practice. Usually, data about a certain phenomena comes from different sources in different times, so we want to combine these data to study such phenomena. Many authors have considered the problem of combining (n) independent tests of hypotheses. For simple null hypotheses, Little and Folks (1971), studied four methods for combining a finite number of independent tests. They found that the Fisher method is better than the other three methods via Bahadur efficiency. Again, Little and Folks (1973) studied all methods of combining a finite number of independent tests and thy found that the Fisher's method is optimal under some mild conditions.

W. A. Abu-Dayyeh, Department of Mathematical Sciences, Dhahran, Saudi Arabia M. MA. Al-Momani, Department of Mathematical Sciences, Dhahran, Saudi Arabia

Z. R. Al-Rawi Chairman, Department of Statistics Yarmouk University, Irbid, Jordan For correspondence regarding this article, send Email to alrawiz@yu.edu.jo. This work was carried out with financial support from the Yarmouk University Research Council.
Brown, Cohen and Strawderman (1976) have shown that such all tests form a complete class. Abu-Dayyeh and Bataineh (1992) showed that the Fisher's method is strictly dominated by the sum of P-values method via Exact Bahadur Slop in case of combining an infinite number of independent shifted exponential tests when the sample size remains finite. Also, Abu-Dayyeh (1992) showed that under certain conditions that the local limit of the ratio of the Exact Bahadur efficiency of two tests equivalent to the Pitman efficiency between the two tests where these tests are based on sum of iid r.v's. Again AbuDayyeh and El-Masri (1994) studied the problem of combining (n) independent tests as $(\mathrm{n} \rightarrow \infty)$ in case of triangular distribution using six methods viz. sum of $\mathrm{P}$-values, inverse normal, logistic, Fisher, minimum of P-values and maximum of P-values. They showed that the sum of P-values is better than all other methods.

Abu-Dayyeh (1997) extended the definition of the local power of tests to the case of having nuisance parameters. He derived the local power for any symmetric test in the case of a bivariate normal distribution with known correlation coefficient, and then he applied it to the combination methods.

Specific Problem

Suppose there is $(n)$ simple hypotheses:

$H_{0}^{(i)}: \theta_{i}=\theta_{0 i} \quad$ vs $\quad H_{1}^{(i)}: \theta_{i}>\theta_{0 i} \quad i=1,2, \ldots, n$ 
Where $\theta_{0 i}$ is known for $i=1,2, \ldots, n$ and $H_{0}^{(i)}$ is rejected for sufficiently large values of some continuous real valued test statistic $T^{(i)}$, $i=1,2, \ldots, n$ and we want to combine the $(n)$ hypotheses into one hypothesis as follows:

VS

$$
H_{0}:\left(\theta_{1}, \theta_{2}, \ldots, \theta_{n}\right)=\left(\theta_{01}, \theta_{02}, \ldots, \theta_{0 n}\right)
$$$$
H_{1}: \theta_{i} \geq \theta_{0 i} \text { for all } \mathrm{i} \text {, and } \theta_{i}>\theta_{0 \mathrm{i}} \text { for }
$$

some $i, i=1,2, \ldots, n$

Many methods have been used for combining several tests of hypotheses into one overall test. Among these methods are the nonparametric (omnibus) methods that combine the $\mathrm{P}$-values of the different tests. The P-value of the $i$ - $t h$ hypothesis is given by:

$$
P_{i}=\underset{H_{0}^{(i)}}{P}\left(T^{(i)} \geq t\right)=1-\underset{H_{0}^{(i)}}{F}(t)
$$

where $F_{H O}{ }^{(\mathrm{i})}(t)$ is the $c d f$ of $T^{(\mathrm{i})}$ under $H_{0}{ }^{(i)}$. Note that $P_{i} \sim U(0,1)$ under $H_{0}{ }^{(i)}$.

Considered in this article is the case of $\theta_{i}^{*}=\gamma \theta_{i}, \quad$ where $\theta_{1}, \theta_{2}, \ldots, \theta_{r} \geq 0$ fixed constants and $\gamma$ is the unknown parameter. Then $T^{(1)}, T^{(2)}, \ldots, T^{(r)}$ are independent $r . v^{\prime} s$ such that for $i=1,2, . ., r$ and we want to test

$$
\begin{aligned}
& H_{0}: \gamma=0 \quad \text { vs } \\
& H_{1}: \gamma>0
\end{aligned}
$$

and therefore considered is the problem of combining a finite number of independent tests by looking at the Local Power of tests which is defined for a test $\varphi$ by:

$$
\mathrm{L}_{\mathrm{P}}(\varphi)=\left.\inf _{\theta} \frac{\partial}{\partial \gamma} \mathrm{E}_{\gamma \theta}(\varphi)\right|_{\gamma=0}
$$

where

$\gamma \geq 0, \theta=\left(\theta_{1}, \theta_{2}, \ldots, \theta_{r}\right), \theta_{i} \geq 0, i=1,2, \ldots, r$, in case of logistic distribution. Compared (5) for the four methods of combining tests for the location family of distributions when $r=2$ and $r=3$. These methods are: Fisher, logistic, the sum of p-values and the inverse normal methods.

\section{Methodology}

Now we will find expressions for the Local Power of the four combination methods of tests then compare them via the Local Power.

Lemma 1

Let $X_{1}, X_{2}$ be independent r.v's such that $X_{i} \sim \operatorname{Logistic}\left(\gamma \theta_{i}, 1\right)$ for $i=1,2$. Then

$\mathrm{A}(1)$

$\left.\frac{\partial}{\partial \gamma} \mathrm{E}_{\gamma\left(\theta_{1}, \theta_{2}\right)}\left(\varphi_{\mathrm{F}}\right)\right|_{\gamma=0}=\mathrm{K}_{\mathrm{F}}\left(\theta_{1}+\theta_{2}\right)$,where

$K_{F}=\int_{1}^{a}\left(1-e^{-c / 2} y\right) \frac{2-y}{y^{3}} d y, \quad a=e^{c / 2} \quad$ and $c=\chi_{(4),(1-\alpha)}^{2}$

$\mathrm{A}(2)$

$\left.\frac{\partial}{\partial \gamma} \mathrm{E}_{\gamma\left(\theta_{1}, \theta_{2}\right)}\left(\varphi_{\mathrm{L}}\right)\right|_{\mathrm{y}=0}=\mathrm{K}_{\mathrm{L}}\left(\theta_{1}+\theta_{2}\right)$, where $K_{L}=\int_{1}^{\infty} \frac{(y-2)(y-1)}{\left(y-1+e^{-c}\right) y^{3}} d y$, and $c$ satisfies the following $1-\alpha=\frac{1-e^{-c}(c+1)}{\left(1-e^{-c}\right)^{2}}$.

$$
\begin{gathered}
\left.\frac{\partial}{\partial \gamma} \mathrm{E}_{\gamma\left(\theta_{1}, \theta_{2}\right)}\left(\varphi_{\mathrm{S}}\right)\right|_{\gamma=0}=\mathrm{K}_{\mathrm{S}}\left(\theta_{1}+\theta_{2}\right) \text {, where } \\
K_{S}=\frac{c^{2}(3-2 c)}{6} \text {, and } c=\sqrt{2 \alpha} .
\end{gathered}
$$


$\left.\frac{\partial}{\partial \gamma} \mathrm{E}_{\gamma\left(\theta_{1}, \theta_{2}\right)}\left(\varphi_{\mathrm{N}}\right)\right|_{\gamma=0}=\mathrm{K}_{\mathrm{N}}\left(\theta_{1}+\theta_{2}\right)$, where

$K_{N}=-\int_{1}^{a}\left(1-\Phi\left(-c-\Phi^{-1}\left(\frac{1}{y}\right)\right)\right) \frac{y-2}{y^{3}} d y$

$a=\frac{1}{\Phi(-c)}$ and $c=\sqrt{2} \Phi^{-1}(1-\alpha)$.

Proofs of the previous lemma are similar to proofs of lemma 2, so we will not write it.

Lemma 2

Let $X_{1}, X_{2}, X_{3}$ be independent r.v's such that $X_{i} \sim \operatorname{Logistic}\left(\gamma \theta_{i}, 1\right)$ for $i=1,2,3$. Then

$\left.\mathrm{B}(1) \frac{\partial}{\partial \gamma} E_{\gamma\left(\theta_{1}, \theta_{2}, \theta_{3}\right)}\left(\varphi_{F}\right)\right|_{\gamma=0}=K_{F} \sum_{i=1}^{3} \theta_{i}$ $=K_{F}\left(\theta_{1}+\theta_{2}+\theta_{3}\right) \quad$ where

$K_{F}=\int_{1}^{a}\left[1-e^{-c / 2} y\left(1+\frac{c}{2}-\ln (y)\right)\right] \frac{y-2}{y^{3}} d y$ , $a=e^{c / 2}$, and $c=\chi_{(6),(1-\alpha)}^{2}$.

${ }^{\prime} \mathrm{B}(2)$

, where

$K_{L}=-\int_{1}^{\infty} \int_{1}^{\infty} \frac{(u-1)(v-1)(2-v)}{(u-1)(v-1)+e^{-c}} \frac{1}{u^{2}} \frac{1}{v^{3}} d u d v$, and $c$ satisfies the following:

$1-\alpha=\int_{1}^{\infty} \int_{1}^{\infty} \frac{(u-1)(v-1)}{(u-1)(v-1)+e^{-c}} \frac{1}{u^{2}} \frac{1}{v^{2}} d u d v$

$$
\begin{aligned}
& \left.\frac{\partial}{\partial \gamma} E_{\gamma\left(\theta_{1}, \theta_{2}, \theta_{3}\right)}\left(\varphi_{S}\right)\right|_{\gamma=0}, \\
& =K_{S} \sum_{i=1}^{3} \theta_{i}=K_{S}\left(\theta_{1}+\theta_{2}+\theta_{3}\right) \text { where } \\
& K_{S}=\frac{c^{3}(2-c)}{12} \text { and } c=\sqrt[3]{6 \alpha} .
\end{aligned}
$$

$\mathrm{B}(4)$

$$
\begin{aligned}
& \left.\frac{\partial}{\partial \gamma} E_{\gamma\left(\theta_{1}, \theta_{2}, \theta_{3}\right)}\left(\varphi_{N}\right)\right|_{\gamma=0}=K_{N} \sum_{i=1}^{3} \theta_{i}, \\
& =K_{N}\left(\theta_{1}+\theta_{2}+\theta_{3}\right)
\end{aligned}
$$

where

$K_{N}=$

$-\int_{1}^{a b} \int_{1}^{b}\left(1-\Phi\left(-c-\Phi^{-1}(u)-\Phi^{-1}(v)\right)\right)(1-2 v) d u d v$

$a=\Phi(-c), \quad b=\Phi\left(-c-\Phi^{-1}(v)\right), \quad$ and $c=\sqrt{3} \Phi^{-1}(1-\alpha)$.

Now, we will prove just $B(1)$, because the proof of the others can be done in the same way.

Proof of $\mathrm{B}(1)$ :

$$
E_{\gamma\left(\theta_{1}, \theta_{2}, \theta_{3}\right)}\left(\varphi_{F}\right)=\int_{-\infty}^{\infty} \int_{-\infty}^{\infty} \int_{-\infty}^{\infty} \phi_{F} \prod_{i=1}^{3} f\left(x_{i}-\gamma \theta_{i}\right) d x_{i},
$$

where $f\left(x_{i}-\gamma \theta_{i}\right)$ is the p.d.f of $\operatorname{Logistic}\left(\gamma \theta_{i}, 1\right)$ for $i=1,2,3$

It easy to show that: $E_{\gamma\left(\theta_{1}, \theta_{2}, \theta_{3}\right)}\left(\varphi_{F}\right)$

$=1-\int_{-\infty}^{\infty} \int_{-\infty}^{\infty} \int_{-\infty}^{\infty}\left(1-\phi_{F}\right) \prod_{i=1}^{3} f\left(x_{i}-\gamma \theta_{i}\right) d x_{i}$ 
78

ABU-DAYYEH, AL-RAWI, \& AL-MOMANI

SO,

$\frac{\partial}{\partial \gamma} E_{\gamma\left(\theta_{1}, \theta_{2}, \theta_{3}\right)}\left(\varphi_{F}\right)$

$=\frac{\partial}{\partial \gamma}\left[1-\int_{-\infty}^{\infty} \int_{-\infty}^{\infty} \int_{-\infty}^{\infty}\left(1-\phi_{F}\right) \prod_{i=1}^{3} f\left(x_{i}-\gamma \theta_{i}\right) d x_{i}\right]$

$\frac{\partial}{\partial \gamma} E_{\gamma\left(\theta_{1}, \theta_{2}, \theta_{3}\right)}\left(\varphi_{F}\right) \mid=-\int_{-\infty}^{\infty} \int_{-\infty}^{\infty} \int_{-\infty}^{\infty}\left(1-\varphi_{F}\right) \times$

$\left\{\begin{array}{l}f\left(x_{1}\right) f\left(x_{2}\right) f^{\prime}\left(x_{3}\right)+f\left(x_{1}\right) f^{\prime}\left(x_{2}\right) f\left(x_{3}\right) \\ +f^{\prime}\left(x_{1}\right) f\left(x_{2}\right) f\left(x_{3}\right)\end{array}\right\}$

$d x_{1} d x_{2} d x_{3}$

such that when

$\gamma=0, f^{\prime}\left(x_{i}\right)=\frac{\theta_{i}\left(e^{-x_{i}}-e^{-2 x_{i}}\right)}{\left(1+e^{-x_{i}}\right)^{3}}$ for $i=1,2,3$.

By symmetric of

$x_{i}$ we have

$\left.\frac{\partial}{\partial \gamma} \mathrm{E}_{\gamma\left(\theta_{1}, \theta_{2}, \theta_{3}\right)}\left(\varphi_{\mathrm{F}}\right)\right|_{\gamma=0}=\left(\sum_{\mathrm{i}=1}^{3} \theta_{\mathrm{i}}\right) \mathrm{K}_{\mathrm{F}}$, where

$K_{F}=$

$-\int_{-\infty}^{\infty} \int_{-\infty}^{\infty} \int_{-\infty}^{\infty}\left(1-\varphi_{F}\right) \frac{e^{-x_{1}}}{\left(1+e^{-x_{1}}\right)^{2}} \frac{e^{-x_{2}}}{\left(1+e^{-x_{2}}\right)^{2}} \frac{\left(e^{-x_{3}}-e^{-2 x_{3}}\right)}{\left(1+e^{-x_{3}}\right)^{3}} d x_{1} d x_{2} d x_{3}$

where $\quad 1-\varphi_{F}=\left\{\begin{array}{l}1,-2 \sum_{i=1}^{3} \ln \left(p_{i}\right) \leq c \\ 0, \quad \text { ow }\end{array}\right.$,

$p_{i}=\frac{1}{1+e^{x_{i}}}, i=1,2,3$

$-2 \ln \left(p_{1}\right)-2 \ln \left(p_{2}\right)-2 \ln \left(p_{3}\right) \leq c$ implies

that $x_{1} \leq \ln \left(\frac{e^{c / 2}}{\left(e^{x_{2}}+1\right)\left(e^{x_{3}}+1\right)}-1\right)$,

also
$-2 \ln \left(p_{2}\right)-2 \ln \left(p_{3}\right) \leq c$ and

$-2 \ln \left(p_{3}\right) \leq c$ implies

that $x_{2} \leq \ln \left(\frac{e^{c / 2}}{\left(e^{x_{3}}+1\right)}-1\right)$

and $x_{3} \leq \ln \left(e^{c / 2}-1\right)$ respectively.

Let $a=\ln \left(e^{c / 2}-1\right)$,

$b=\ln \left(\frac{e^{c / 2}}{\left(e^{x_{3}}+1\right)}\right)$ and let

$d=\ln \left(\frac{e^{c / 2}}{\left(e^{x_{2}}+1\right)\left(e^{x_{3}}+1\right)}-1\right)$, then we will

get

$K_{F}=$

$-\int_{-\infty}^{a} \int_{-\infty-\infty}^{d} \int_{-\infty}^{d} \frac{e^{-x_{1}}}{\left(1+e^{-x_{1}}\right)^{2}} \frac{e^{-x_{2}}}{\left(1+e^{-x_{2}}\right)^{2}} \frac{\left(e^{-x_{j}}-e^{-2 x_{5}}\right)}{\left(1+e^{-x_{j}}\right)^{3}} d x_{1} d x_{2} d x_{3} \cdot$

Let $I_{1}=\int_{-\infty}^{d} \frac{e^{-x_{1}}}{\left(1+e^{-x_{1}}\right)^{2}} d x_{1}$, then put

$u=1+e^{-x_{1}}$ to get that $I_{1}=\frac{1}{1+e^{-d}}$,

so, $I_{1}=1-e^{-c / 2}\left(e^{x_{2}}+1\right)\left(e^{x_{3}}+1\right)$.

$\therefore K_{F}=$

$-\int_{-\infty-\infty}^{a} \frac{e^{-x_{2}}}{\left(1+e^{-x_{2}}\right)^{2}} \frac{\left(e^{-x_{5}}-e^{-2 x_{x}}\right)}{\left(1+e^{-x_{5}}\right)^{3}}\left(1-e^{-c / 2}\left(e^{x^{5}}+1\right)\left(e^{x_{5}}+1\right)\right) d x_{1} d x_{2}$

Also,

$I_{2}=\int_{-\infty}^{b} \frac{e^{-x_{2}}}{\left(1+e^{-x_{2}}\right)^{2}}\left(1-e^{-c / 2}\left(e^{x_{2}}+1\right)\left(e^{x_{3}}+1\right)\right) d x_{2}$ 


$$
\begin{gathered}
=\int_{-\infty}^{b} \frac{e^{-x_{2}}}{\left(1+e^{-x_{2}}\right)^{2}} d x_{2} \\
-e^{-c / 2}\left(e^{x_{3}}+1\right) \int_{-\infty}^{b} \frac{1}{\left(1+e^{-x_{2}}\right)} d x_{2} \\
=1-e^{-c / 2}\left(e^{x_{3}}+1\right) \\
-e^{-c / 2}\left(e^{x_{3}}+1\right)\left(\frac{c}{2}-\ln \left(e^{x_{3}}+1\right)\right) \\
=1-e^{-c / 2}\left(e^{x_{3}}+1\right)\left(1+\frac{c}{2}-\ln \left(e^{x_{3}}+1\right)\right) \\
\left.\left.\therefore K_{F}=\frac{c}{a}-\ln \left(e^{x_{3}}+1\right)\right)\right] d x_{3} . \\
-\int_{-\infty}^{a} \frac{e^{-x_{3}}-e^{-2 x_{3}}}{\left(1+e^{-x_{3}}\right)}\left[1-e^{-c / 2}\left(e^{x_{3}}+1\right)\left(1+\frac{1}{2}\right)\right.
\end{gathered}
$$

Finally put $y=1+e^{x_{3}}$ we get

$$
K_{F}=\int_{1}^{d}\left(1-e^{-c / 2} y\left(1+\frac{c}{2}-\ln (y)\right)\right) \frac{y-2}{y^{3}} d y,
$$$$
d=e^{c / 2}
$$$$
\alpha=P_{0}\left(-2 \sum_{i=1}^{3} \ln \left(p_{i}\right) \geq c\right)=1-P_{0}\left(-2 \sum_{i=1}^{3} \ln \left(p_{i}\right) \leq c\right),
$$

because $-2 \sum_{i=1}^{3} \ln \left(p_{i}\right) \sim \chi_{(6)}^{2}$

under $H_{0}$, then $c=\chi_{(6),(1-\alpha)}^{2}$, which completes the proof.

Also, here for the logistic distribution we will compare the Local Power for the previous four tests numerically. So from tables (1) and (2) when $\alpha=0.01$ and $r=2$ the sum of p-values method is the best method followed by the inverse normal method, the logistic method and Fisher method respectively, but for all of the other values of $\alpha$ and $r$ the inverse normal method is the best method followed by the sum of p-values method followed by logistic method and the worst method is Fisher method.

\section{References}

Abu-Dayyeh, W. A. (1989). Bahadur exact slope, pitman efficiency and local power combining independent tests. Ph.D. Thesis, University of Illinois at Urbana - Champaign.

Abu-Dayyeh, W. A. (1992). Exact bahadur efficiency. Pakistan Journal of Statistics, 8 (2), 53-61.

Abu-Dayyeh, W. A., \& Bataineh (1992). Comparing the exact Bahadur of the Fisher and sum of P-values methods in case of shifted exponential distribution. Mu'tah slopes. Journal for Research and Studies, 8, 119-130.

Abu-Dayyeh, W. A., \& El-Masri. (1994). Combining independent tests of triangular distribution. Statistics \& Probability Letters, 21, 195-202.

Abu-Dayyeh, W. A. (1997). Local power of tests in the prescience of nuisance parameters with an application. The Egyptian Statistical Journal ISSR, 41, 1-9.

Little, R. C., \& Folks, L. J. (1971). Asymptotic optimality of Fisher's method of combining independent tests. Journal of the American Statistical Association, 66, 802-806.

Little, R. C., \& Folks, L. J. (1973). Asymptotic optimality of Fisher's method of combining Independent Tests II. Journal of the American Statistical Association, 68, 193-194. 
The following tables explain the term $K_{A}$ where $A \in\{F, L, S, N\}$ for the logistic distributions.

Table (1): Local power for the logistic distribution when $(r=2)$

\begin{tabular}{|c|c|c|c|c|}
\hline$\alpha$ & $K_{F}$ & $K_{L}$ & $K_{S}$ & $K_{N}$ \\
\hline 0.010 & 0.0073833607 & 0.0081457298 & 0.0090571910 & 0.0089064740 \\
\hline 0.025 & 0.0174059352 & 0.0192749938 & 0.0212732200 & 0.0214554551 \\
\hline 0.050 & 0.0326662436 & 0.0361783939 & 0.0394590744 & 0.0415197403 \\
\hline
\end{tabular}

Table (2): Local power for the logistic distribution when $(r=3)$

\begin{tabular}{|c|c|c|c|c|}
\hline$\alpha$ & $K_{F}$ & $K_{L}$ & $K_{S}$ & $K_{N}$ \\
\hline 0.010 & 0.0062419188 & 0.0071070250 & 0.0080425662 & 0.0083424342 \\
\hline 0.025 & 0.0144747833 & 0.0165023359 & 0.0183583839 & 0.0199610766 \\
\hline 0.050 & 0.0267771426 & 0.0304639648 & 0.0332641762 & 0.0381565019 \\
\hline
\end{tabular}

